\title{
Ebola drug trial is terminated after fall in number of new cases
}

\author{
Anne Gulland
}

London

Researchers have announced that they are stopping the trial of an experimental Ebola virus drug because of a significant drop in the number of new cases of infection.

The trial, led by scientists from the University of Oxford at a Médecins Sans Frontières (MSF) treatment centre in Liberia, began on 2 January, but the drug's manufacturer unexpectedly announced on 30 January that it was withdrawing from the trial.

Chimerix, the US company that manufactured the drug brincidofovir, said that only a handful of patients had been enrolled in the study; researchers had initially hoped to enrol about 100 patients.

The termination of the trial will disappoint the researchers, who managed to pull together a clinical trial in four months instead of the usual 18 months. ${ }^{1}$ Peter Horby, lead investigator on the trial, said, "The past weeks have brought the extremely positive news that Ebola infections are falling across west Africa, including in Liberia where our trial of brincidofovir was based.

"We're delighted that infections are falling, but fewer patients makes it more difficult to carry out the robust scientific studies needed to ensure a new treatment will be safe and effective."

In the week to 25 January the World Health Organization reported just four new cases of Ebola virus disease in Liberia, down from 300 new cases a week in August and September last year. The number of new cases is also falling in Guinea and Sierra Leone.

Jeremy Farrar, director of the Wellcome Trust, which is providing $£ 3.2 \mathrm{~m}$ ( $€ 4.3 \mathrm{~m} ; \$ 4.9 \mathrm{~m}$ ) to fund this and other Ebola drug trials, said that it was disappointing that the trial could not continue. But he added, "It is essential that other studies of potential treatments and vaccines continue and hopefully will still be able to deliver meaningful results for this and the inevitable future epidemics."

MSF said that low patient numbers were also proving a challenge for the other trials taking place at its treatment centres. A trial of the antiviral drug favipiravir is taking place at one of its centres in Guinea, and a trial using plasma from recovered Ebola patients will start next week.

Bertrand Draguez, MSF medical director, said, "The number of patients may be declining but the epidemic certainly isn't over yet... We remain dedicated to finding out as much as possible about potential treatments, vaccines, and diagnostic tools to prevent a repetition of this tragic loss of lives."

1 Gulland A. Trials of Ebola vaccine set to begin in west Africa. BMJ 2015;350:h165. 